\title{
Efficacy and Safety of All-oral, 12-week Ravidasvir Plus Ritonavir-boosted Danoprevir and Ribavirin in Treatment-naïve Noncirrhotic HCV Genotype 1 Patients: Results from a Phase 2/3 Clinical Trial in China
}

Xiaoyuan $\mathrm{Xu}^{1}$, Bo Feng ${ }^{2}$, Yujuan Guan ${ }^{3}$, Sujun Zheng ${ }^{4}$, Jifang Sheng ${ }^{5}$, Xingxiang Yang ${ }^{6}$, Yuanji Ma ${ }^{7}$, Yan Huang ${ }^{8}$, Yi Kang ${ }^{9}$, Xiaofeng Wen ${ }^{10}$, Jun $\mathrm{Li}^{11}$, Youwen $\operatorname{Tan}^{12}$, Qing He ${ }^{13}$, Qing Xie ${ }^{14}$, Maorong Wang ${ }^{15}$, Ping An ${ }^{16}$, Guozhong Gong ${ }^{17}$, Huimin Liu ${ }^{18}$, Qin Ning ${ }^{19}$, Rui Hua ${ }^{20}$, Bo Ning ${ }^{21}$, Wen Xie ${ }^{22}$, Jiming Zhang ${ }^{23}$, Wenxiang Huang ${ }^{24}$, Yongfeng Yang ${ }^{25}$, Minghua Lin ${ }^{26}$, Yingren Zhao ${ }^{27}$, Yanhong $\mathrm{Yu}^{28}$, Jidong Jia ${ }^{29}$, Dongliang Yang ${ }^{30}$, Liang Chen ${ }^{31}$ Yinong $\mathrm{Ye}^{32}$, Yuemin $\mathrm{Nan}^{33}$, Zuojiong Gong ${ }^{34}$, Quan Zhang ${ }^{35}$, Peng Hu ${ }^{36}$, Fusheng Wang ${ }^{37}$ ', Yongguo $\mathrm{Li}^{38}$, Dongliang $\mathrm{Li}^{39}$, Zhansheng Jia ${ }^{40}$, Jinlin Hou ${ }^{41}$, Chengwei Chen ${ }^{42}$, Jinzi J. Wu ${ }^{43}$ and Lai Wei*44

\begin{abstract}
${ }^{1}$ Peking University First Hospital, Beijing, China; ${ }^{2}$ Peking University People's Hospital, Beijing, China; ${ }^{3}$ Guangzhou Eighth People's Hospital, Guangzhou, China; ${ }^{4}$ Beijing YouAn Hospital, Capital Medical University, Beijing, China; ${ }^{5}$ The First Affiliated Hospital of Medical School of Zhejiang University, Hangzhou, China; ${ }^{6}$ Sichuan Provincial People's Hospital, Chengdu, China; ${ }^{7}$ West China Hospital, Sichuan University, Chengdu, China; ${ }^{8}$ Xiangya Hospital, Central South University, Changsha, China; ${ }^{9}$ Henan Provincial People's Hospital, Zhengzhou, China; ${ }^{10}$ Liuzhou People's Hospital, Liuzhou, China; ${ }^{11}$ Jiangsu Province Hospital, Nanjing, China;

${ }^{12}$ Zhenjiang No.3 People's Hospital, Zhenjiang, China; ${ }^{13}$ The Third People's Hospital of Shenzhen, Shenzhen, China; ${ }^{14}$ Ruijin Hospital, Shanghai Jiaotong University School of Medicine, Shanghai, China; ${ }^{15}$ People's Liberation Army Bayi Hospital, Nanjing, China; ${ }^{16}$ Shenyang Sixth People's Hospital, Shenyang, China; ${ }^{17}$ The 2nd Xiangya Hospital of Central South University, Changsha, China; ${ }^{18}$ Xixi Hospital of Hangzhou, Hangzhou, China; ${ }^{19}$ Tongji Medical College of Huazhong University of Science \& Technology, Wuhan, China; ${ }^{20}$ The First Hospital of Jilin University, Changchun, China; ${ }^{21}$ Baoji Center Hospital, Baoji, China; ${ }^{22}$ Beijing Ditan Hospital, Beijing, China; ${ }^{23}$ Huashan Hospital Affiliated to Fudan University, Shanghai, China; ${ }^{24}$ Chongqing Medical University No.1 Affiliated Hospital, Chongqing, China; ${ }^{25}$ The Second Hospital of Nanjing, Nanjing, China; ${ }^{26}$ Fujian Fuzhou Municipal Infectious Disease Hospital, Fuzhou, China; ${ }^{27}$ No. 1 Affiliated Hospital of Xi'an Jiaotong University, Xi'an, China; ${ }^{28}$ The First Affiliated Hospital of Guangxi Medical University, Nanning, China; ${ }^{29}$ Beijing Friendship Hospital, Capital Medical University, Beijing, China; ${ }^{30}$ Wuhan Union Hospital, Wuhan, China; ${ }^{31}$ Shanghai Public Health Clinical Center, Shanghai, China; ${ }^{32}$ Foshan No.1 People's Hospital, Foshan, China; ${ }^{33}$ The Third Hospital of Hebei Medical University, Shijiazhuang, China; ${ }^{34}$ Wuhan University Renmin Hospital, Wuhan, China; ${ }^{35}$ The Affiliated Hospital of Guizhou Medical University, Guiyang, China; ${ }^{36}$ The Second Affiliated Hospital of Chongqing Medical University, Chongqing, China; ${ }^{37}$ PLA 302 Hospital, Beijing, China; ${ }^{38}$ The First Affiliated Hospital of Harbin Medical University, Harbin, China; ${ }^{39}$ Fuzhou General Hospital of Nanjing Military Command, Fuzhou, China; ${ }^{40}$ Tang Du Hospital, Fourth military Medical University, Xi'an, China; ${ }^{41}$ Nanfang Hospital, Nanfang Medical University, Guangzhou, China; ${ }^{42}$ The 85 branch of the Chinese People's Liberation Army Hospital, Shanghai, China; ${ }^{43}$ Ascletis BioScience Co., Ltd. Hangzhou, China;

${ }^{44}$ Tsinghua Changgeng Hospital, Beijing, China
\end{abstract}

\section{Abstract}

Background and Aims: Ravidasvir (RDV) is a new generation pangenotypic hepatitis C virus (HCV) NS5A inhibitor, with high barrier to baseline resistance-associated species. This is the first phase $2 / 3$ study conducted in Mainland China

Keywords: Ravidasvir; Danoprevir; HCV; SVR; Treatment-naïve; Noncirrhotic; GT1.

Abbreviations: AE, adverse event; ALT, alanine aminotransferase; AST, aspartate aminotransferase; CI, confidence interval; DAA, direct acting antiviral therapy; DNVr, ritonavir-boosted danoprevir; GT1, genotype $1 ; \mathrm{HCV}$, hepatitis $\mathrm{C}$ virus; RBV, ribavirin; RDV, ravidasvir; SAE, serious adverse event; SVR12, sustained virological response at 12 weeks after treatment.

Received: 30 July 2019; Revised: 12 September 2019; Accepted: 15 September 2019

*Correspondence to: Lai Wei, Tsinghua Changgeng Hospital, No. 168 Li Tang Road, Chang Ping District, Beijing 102218, China. Tel: +86-10-88326666, Fax: +86-10-68318386, E-mail: weelai@163.com confirming the efficacy and safety of RDV + ritonavir-boosted danoprevir + ribavirin for 12 weeks in treatment-naïve noncirrhotic patients with genotype 1 infection in a large population. Methods: In this multicenter, randomized, doubleblinded, placebo-controlled phase 2/3 trial (NCT03362814), we enrolled 424 treatment-naïve, noncirrhotic adult HCV genotype 1 patients. All patients were randomized at $3: 1$ ratio to receive a combination of RDV $200 \mathrm{mg}$ once daily plus ritonavir-boosted danoprevir $100 \mathrm{mg} / 100 \mathrm{mg}$ twice daily and oral ribavirin $1000 / 1200 \mathrm{mg} /$ day (body weight $<75 / \geq 75 \mathrm{~kg}$ ) $(n=318)$ or placebo $(n=106)$ for 12 weeks. The primary end-point was the rate of sustained virologic response 12 weeks after the end of treatment, and the safety was evaluated and compared between treatment and placebo groups. Results: The overall rate of sustained virological response at 12 weeks after treatment is 99\% (306/309, $95 \%, \mathrm{CI}: 97 \%-100 \%$ ) under per protocol set analysis. 
Xu X. et al: Efficacy of RDV + DNV regimen in GT1 CHC patients

All patients harboring baseline NS5A resistance-associated species in the treatment group (76/76, per protocol set) achieved sustained virological response at 12 weeks after treatment. No treatment-related serious adverse events were reported. Laboratory abnormalities showed mild or moderate severity (grade 1 and grade 2 ) in liver function tests. Conclusions: In treatment-naïve, noncirrhotic HCV Chinese patients infected with HCV genotype 1, all-oral regimen of RDV + ritonavir-boosted danoprevir + ribavirin for 12 weeks was highly efficacious, safe, and well tolerated.

Citation of this article: Xu X, Feng B, Guan Y, Zheng S, Sheng J, Yang $X$, et al. Efficacy and safety of all-oral, 12-week ravidasvir plus ritonavir-boosted danoprevir and ribavirin in treatment-naïve noncirrhotic HCV genotype 1 patients: Results from a phase $2 / 3$ clinical trial in China. J Clin Transl Hepatol 2019;7(3):213-220. doi: 10.14218/JCTH.2019.00033.

\section{Introduction}

In China, more than 10 million people are estimated to be chronically infected with hepatitis C virus (HCV), ${ }^{1}$ with genotype 1 (GT1) b as the most prevalent sub-genotype $(56.8 \%) .^{2}$ Currently, approximately $50-75 \%$ of the patients infected with GT1 HCV are able to achieve a sustained virological response (SVR) after treatment with a combination of pegylated-interferon plus ribavirin (RBV) for 48 weeks, the current standard of care for chronic HCV infection in China, ${ }^{3}$ leaving strong needs for new direct acting anti-viral therapy (DAA) with higher efficacy as warranted.

Ravidasvir (RDV), a potent pangenotypic NS5A inhibitor, has a favorable pharmacokinetic profile, with rapid absorption, stable plasma distribution and high plasma trough concentration, allowing for continuous HCV inhibition with once-daily oral dosing. ${ }^{4}$ Danoprevir (DNV) is a potent, macrocyclic inhibitor of the HCV NS3/4A serine protease ${ }^{5,6}$ that can be co-administered with low-dose ritonavir to achieve therapeutic trough concentrations throughout the dosing interval. Combination of RDV and DNV may serve as a potent regimen against HCV infection.

As reported in the phase 2 EVEREST study, all-oral RDV + ritonavir-boosted danoprevir (DNVr) + RBV regimen has demonstrated sustained virological response at 12 weeks after treatment (SVR12) at $100 \%(38 / 38)$ in treatmentnaïve noncirrhotic Taiwanese patients with HCV GT1 infection, and was safe and well tolerated. ${ }^{7}$ This phase $2 / 3$ study was designed to investigate the safety and efficacy of this combination regimen in treatment-naïve HCV GT1 patients without cirrhosis in a larger population from Mainland China.

\section{Methods}

\section{Patients}

Patients with confirmed chronic HCV infection were screened and recruited from 42 sites in different provinces across China, and eligible subjects were enrolled in the study according to the inclusion and exclusion criteria as below:

\section{Inclusion criteria}

Chinese adult of $\geq 18$ years-old with chronic GT1 HCVinfection; absence of cirrhosis (defined as a liver stiffness measurement $<9.6 \mathrm{kPa}$ by Fibroscan ${ }^{\circledR}$ or a screening liver biopsy indicating no cirrhosis [metavir score $\leq 3$ ]); plasma HCV RNA $\geq 1 \times 10,000 \mathrm{IU} / \mathrm{mL}$; and never received antiviral treatment for $\mathrm{HCV}$ infection.

\section{Exclusion criteria}

Mixed HCV genotypes; HCV genotypes other than GT-1; HBV or HIV co-infection; alpha-fetoprotein $>100 \mathrm{ng} / \mathrm{mL}$; presence or suspected of other chronic liver illness or hepatocellular carcinoma; presence of cirrhosis as assessed by Fibroscan ${ }^{\circledR}$ $(>12.9 \mathrm{kPa}$ ) or liver biopsy (metavir score at 4); body mass index $<18$ or $\geq 30 \mathrm{~kg} / \mathrm{m}^{2}$; anti-HAV (IgM), HBsAg, anti-HEV (IgM) or anti-HIV positivity; ANC $<1.5 \times 10^{9} / \mathrm{L}$; platelet $<100 \times 10^{9} / \mathrm{L}$; hemobilirubin $<110 \mathrm{~g} / \mathrm{L}$ (female) or $<120 \mathrm{~g} / \mathrm{L}$ (male); international normalized ratio $>1.5$; alanine aminotransferase (ALT) or aspartate aminotransferase (AST) $\geq 5 *$ ULN; total bilirubin $\geq 2 * U L N$ (direct bilirubin $\geq 35 \%$ total bilirubin ); or creatinine $\geq 1.5 * U L N$.

\section{Study design}

Eligible subjects were enrolled and randomly assigned at a $3: 1$ ratio to active treatment (the treatment group) or placebo (the placebo group) arms (Fig. 1). During the double-blinded phase, subjects in the treatment arm received a combination of RDV at $200 \mathrm{mg}$ once daily plus DNVr at $100 \mathrm{mg} / 100 \mathrm{mg}$ twice daily and oral RBV at $1000 / 1200 \mathrm{mg} /$ day $(1000 \mathrm{mg}$ daily if the body weight was $<75 \mathrm{~kg}$ and $1200 \mathrm{mg}$ daily if the body weight was $\geq 75 \mathrm{~kg}$ ) for 12 weeks. Subjects in the placebo arm received placebo during the double-blinded phase and entered into a deferred open-labelled active treatment arm for another 12 weeks. All subjected were followed up for 24 weeks after the end of active treatment (Fig. 1).

\section{Study oversight}

All the subjects provided written informed consent. The protocol of this study conformed to the International Conference on Harmonisation guidelines, applicable regulations, and ethical guidelines of the Declaration of Helsinki. The research was approved by the ethical committee of each participating site and was registered at clinicaltrials.gov (trial registration: NCT03362814). All authors had full access to the database and vouch for the completeness and accuracy of the data and analyses as presented.

\section{Efficacy assessments}

Vital signs were measured and samples were collected and stored at screening and on study visits at weeks $1,2,4,8$, and 12 and at post-treatment weeks 4,12 and 24 for analyses biochemistry, hematology, viral load, and urinalysis.

The lower limit of quantitation of HCV RNA was defined as $15 \mathrm{IU} / \mathrm{mL}$, which was determined by using CAP/CTM HCV 2.0 assay. The primary end-point was SVR12 after the end of the treatment. Viral breakthrough was defined as a one log increase in HCV RNA from postbaseline nadir, or a confirmed positivity in HCV RNA from undetectable level. Post-treatment relapse was defined as HCV RNA returning positivity from previously achieved HCV RNA negativity before or at end of treatment.

We performed resistance analysis by using population sequencing on samples obtained at baseline, when patients had experienced virologic breakthrough during treatment, or 
Xu X. et al: Efficacy of RDV + DNV regimen in GT1 CHC patients

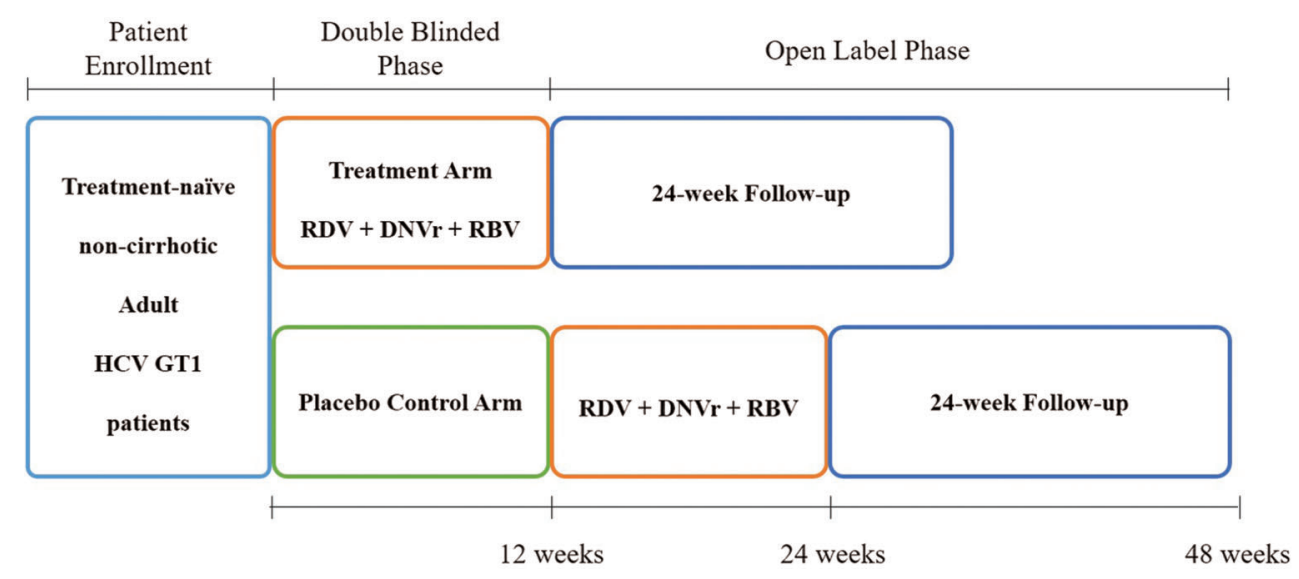

Fig. 1. Study design.

Abbreviations: RDV, ravidasvir; DNVr, ritonavir-boosted danoprevir; RBV, ribavirin.

relapse during follow up after the treatment, in order to evaluate the role and impact from substitutions potentially rendering resistance to NS5A or NS3/4A inhibitors.

\section{Safety assessment}

Adverse events (AEs) were assessed at each study visit and documented for all patients who received at least one dose of medications. Data for all treatment-emergent AEs, laboratory abnormalities, clinically significant changes of vital signs and electrocardiogram testing were obtained from the start of drug administration until 4 weeks after the end of study. AEs were categorized using the Medical Dictionary for Regulatory Activities.

\section{Statistical comparison groups and subgroups}

Analysis population included the FAS subgroup of all randomized subjects who received at least one dose of the study drug and provided at least one point of data; the per protocol population of patients from FAS subjects who met the inclusion criteria without reasons for exclusion and completed the study treatment and follow-up; the safety population of all randomized subjects who received at least one dose of the study drug and provided safety data for appraisal.

\section{Statistical methods}

Demographics and baseline characteristics were collected for the FAS population. Efficacy analyses were performed on the FAS and per protocol set populations, and safety analyses were performed on the per protocol set population. For the primary efficacy end-point (SVR12), a two-sided 95\% confidence interval (CI) was calculated using the normal approximation to the binomial distribution. Subgroup efficacy analyses of SVR12 (including stratification by genotype, baseline HCV RNA level, IL28B genotypes, and baseline polymorphisms in NS5A) were performed for the per protocol set population.

To evaluate the superiority of the RDV plus DNVr and RBV regimen to the historical control in HCV GT1 patients, the lower boundary of the $95 \%$ CI for SVR12 in the treatment and placebo group entering deferred active treatment had to exceed the calculated historical control rate of $75 \%$. The control rate, which was based on response rates among previously untreated noncirrhotic patients who received pegylated-interferon/RBV, was determined from a metaanalysis and experts' clinical experience.

All statistical tests and $95 \%$ CIs were two-sided, and $p$ values $<0.05$ were considered statistically significant. Descriptive statistics for quantitative data used are reported as mean \pm standard deviation and median (interquartile range), while qualitative data is summarized as counts and percentages. Data analysis was performed using SAS Proprietary Software 9.4 (Licensed to PEKING UNIVERSITY HEALTH SCIENCE CENTER, Site 11202165).

\section{Results}

\section{Baseline characteristics and demographics}

A total of 709 subjects were screened, and 425 qualified patients were assigned to randomization. Among whom, 424 (318 patients in the treatment arm and 106 in the placebo arm) received at least one dose of investigational medication and were included in the FAS population for the primary efficacy and safety analyses. A total of 309 patients in the treatment arm and 101 patients in the placebo arm completed the 12-week follow-up and fulfilled the inclusion criteria for the per protocol population (Fig. 2). The demographic and baseline characteristics of the study population are shown in Table 1.

\section{Efficacy}

Overall, the SVR12 rate in the FAS analysis was 96\% (306/ $318,95 \%$ CI, 94 98\%) in the treatment arm and 95\% (101/ $106,95 \% \mathrm{CI}, 93 \sim 100 \%)$ in the placebo arm. In the per protocol population, the SVR12 reached 99\% (306/309, 95\% CI, 97 100\%) in the treatment arm and 99\% (100/101, 95\% CI, 95 100\%) in the placebo arm (Table 2).

All subjects receiving RDV + DNVr + RBV had a rapid decline of serum HCV RNA levels (Fig. 3). By week 4 during the double-blinded treatment phase, the HCV RNA level was below the lower limit of quantitation $(15 \mathrm{IU} / \mathrm{mL}$ ) in $98 \%$ of the patients who were treated by the active regimen ( 303 of the 309 patients in the treatment group), and at the end of the treatment the proportion reached 99\% (306/309). 


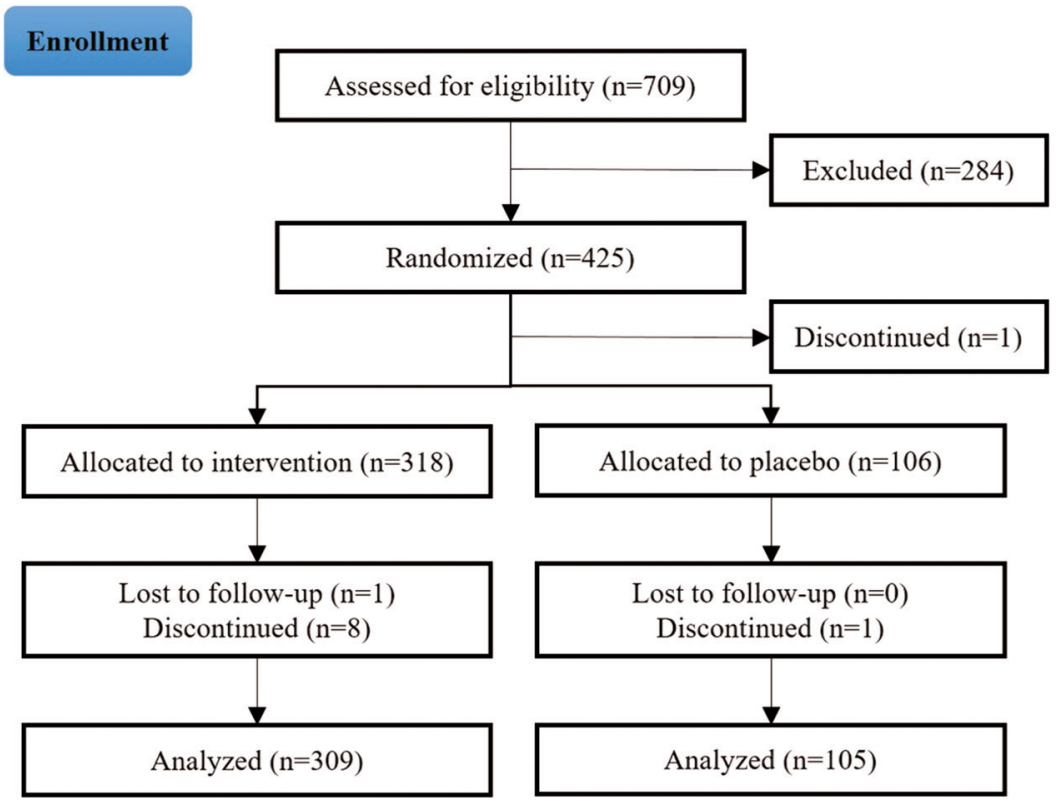

Fig. 2. Consolidated Standards of Reporting Trials (known as CONSORT) flow diagram showing participants flow through each stage of the randomized controlled trial (enrollment, intervention allocation, follow-up, and data analysis). Two hundred and eighty-four patients who failed to meet the standards were excluded from this clinical study and among 425 randomized patients, one voluntarily discontinued without being administered medication. One patient from the placebo arm discontinued due to relocation overseas. Eight patients from the intervention arm discontinued and only one was considered due to an adverse event related to the active treatment.

The SVR12 subanalysis in various subgroups showed that the SVR12 rates were similarly high in all subgroups, including those defined by gender ( $99 \%$ with male and $99 \%$ with female, per protocol set), IL28B genotypes (99\% with CC and $100 \%$ with non-CC, per protocol set), age ( $99 \%$ among people $<65$ years-old and $100 \%$ among people $\geq 65$ yearsold), and initial baseline HCV RNA levels $(100 \%$ with a level $<400,000 \mathrm{IU} / \mathrm{mL}, 97 \%$ with a level of $400,000 \sim 800,000$ $\mathrm{IU} / \mathrm{mL}, 99 \%$ with a level $\geq 800,000 \mathrm{IU} / \mathrm{mL}$ ).

\section{Resistance analyses}

NS5A resistance-associated species were detected in 76 patients $(25 \%, 76 / 309)$ in the treatment arm and 29 subjects in the placebo-controlled arm (29\%, 29/101, per protocol set) at baseline (for details of the resistance-associated species see Table 3). The most common NS5A resistance-associated species detected were R30Q (38/309, 12.3\%), Y93H (21/ $309,6.8 \%)$ and $\mathrm{R} 30 \mathrm{Q}+\mathrm{Y} 93 \mathrm{H}(8 / 309,2.6 \%)$ in the treatment arm and R30Q (21/101, 20.7\%), Y93H (6/101, 5.9\%) and $\mathrm{R} 30 \mathrm{Q}+\mathrm{Y} 93 \mathrm{H}(1 / 101,0.99 \%)$ in the placebo-controlled arm. All patients with baseline NS5A resistance-associated species from both arms achieved SVR12 (Fig. 4).

\section{Treatment failure}

Among 309 patients in the treatment arm, the 76 patients reported with baseline NS5A resistance-associated species all

Table 1. Baseline characteristics and demographic data of subjects

\begin{tabular}{llll}
\hline Parameter & Treatment arm, $n=318$ & Placebo arm, $n=106$ & $p$ value \\
\hline Female, $n(\%)$ & $165(52 \%)$ & $58(55 \%)$ & 0.613 \\
Age, median (range), years & $48(21-73)$ & $45(23-72)$ & 0.01 \\
BMI, mean $\pm \mathrm{SD}, \mathrm{kg} / \mathrm{m}^{2}$ & $23.2 \pm 2.8$ & $22.8 \pm 2.8$ & 0.192 \\
HCV RNA (log10 IU/mL), median & 6.31 & 6.22 & 0.475 \\
$<400,000 \mathrm{IU} / \mathrm{mL}, n(\%)$ & $53(16.67 \%)$ & $21(19.81 \%)$ & $13(12.26 \%)$ \\
$400,000 \mathrm{IU} / \mathrm{mL}-800,000 \mathrm{IU} / \mathrm{mL}, n(\%)$ & $32(10.06 \%)$ & $72(67.92 \%)$ & 0.569 \\
$\geq 800,000 \mathrm{IU} / \mathrm{mL}, n(\%)$ & $233(73.27 \%)$ & $89(84 \%)$ & 0.559 \\
IL28B CC genotype, $n(\%)$ & $259(81 \%)$ & $2(2 \%)$ & 1 \\
HCV genotype $1 \mathrm{a}, n(\%)$ & $6(2 \%)$ & $104(98 \%)$ & 1 \\
HCV genotype $1 \mathrm{~b}, n(\%)$ & $312(98 \%)$ & \\
\hline
\end{tabular}

Abbreviations: BMI, body mass index; HCV, hepatitis $\mathrm{C}$ virus. 
Xu X. et al: Efficacy of RDV + DNV regimen in GT1 CHC patients

Table 2. Sustained virologic response at post-treatment week 12 among all patients after receiving the active regimen

\begin{tabular}{llllllr}
\hline Group & Subgroup & Response/total & SVR $12^{*}$ & $95 \%$ CI & Historical control with PR & $p$ value \\
\hline Treatment arm & FAS & $306 / 318$ & $96 \%$ & $(94 \%, 98 \%)$ & $75 \%$ & $<0.001$ \\
& per protocol set & $306 / 309$ & $99 \%$ & $(97 \%, 100 \%)$ & $75 \%$ & $<0.001$ \\
Placebo arm & FAS & $101 / 106$ & $95 \%$ & $(93 \%, 100 \%)$ & $75 \%$ & $<0.001$ \\
& & $100 / 101$ & $99 \%$ & $(95 \%, 100 \%)$ & $75 \%$ & $<0.001$ \\
\hline
\end{tabular}

*SVR12, sustained response rate at post-treatment week 12 was defined as HCV RNA <15 IU/mL (lower limit of quantitation).

(76/76, per protocol set) achieved SVR12 showing potency of this regimen. Three patients $(0.97 \%)$ experienced a virologic breakthrough during the treatment phase, and no patient relapsed by post-treatment week 12 . Serum samples from two of the three subjects who experienced virologic breakthrough showed positivity for resistance-associated species that confer resistance to both NS3/4A and NS5A. The resistance-associated species were Q80K, V36M, R155K in the protein NS3/4A, and Q30K, Q30H, H58D in the region NS5A. The third patient with virologic breakthrough had no detectable RAS at both baseline and on rise, but the genotype turned out as genotype-2a instead of GT1b as initially diagnosed. This case could not be ruled out from potential mechanisms such as a misdiagnosed genotype on enrollment, viral superinfection with GT1b dominance, or reinfection with genotype-2a during treatment. In the placebo arm, one subject relapsed and showed resistance-associated species R155K in the NS3/4A region and L31V in the NS5A region.

\section{Safety and tolerability}

During the double-blinded phase, $94 \%$ of the subjects in the treatment arm (298/318), experienced adverse events (AEs) compared to $79 \%$ of those in the placebo arm (84/106). The majority of AEs were mild. Only 15 of the 318 subjects in the treatment arm $(15 / 318,5 \%)$ and 9 subjects $(9 / 103,9 \%)$ in the placebo arm had grade 3 treatment-related AEs. The laboratory abnormality tests revealed evaluated liver functions (ALT, AST, total bilirubin, direct bilirubin) in the treatment arm were mostly of grade 1 or 2 and no grade 4 AEs occurred. No treatment-related severe AEs or death occurred during the full treatment course. The most common AEs reported were anemia (in $40 \%$ of the subjects in the treatment arm and 5\% in the placebo arm) and upper respiratory tract infection (in $21 \%$ of the subjects in the treatment arm and $21 \%$ in the placebo arm). Hemobilirubin elevation (16\% vs. $6 \%$ ) was also seen among the patients with the most frequent treatment-related AEs that occurred but was in $<10 \%$ of subjects (Table 4 ).

Discontinuation due to any AE occurred in one patient in the treatment arm, owing to drug allergy. One death was reported due to traffic accident but was not considered treatmentrelated. Most of the liver function abnormalities were mild or moderate (grade 1 or 2 ), and no grade 4 abnormalities were observed. In the treatment arm, four of the three hundredeighteen patients had grade 3 treatment-related elevated total bilirubin level and one had grade 3 elevated ALT. In the placebo arm, three patients experienced grade 3 ALT elevation and two with AST elevation, respectively.

\section{Discussion}

A cross-sectional observational study conducted in 2014 in China revealed HCV GT1b to be the most prevalent subtype at $56.8 \%$ and to be associated with low response to traditional treatment with pegylated-interferon plus RBV for 48 weeks. Recently, some DAA regimens were approved in China but with pre-requisition according to results from a baseline resistance-associated species test. Baseline resistance-associated species in HCV are somehow prevalent and may pose a necessity of intervention in either diagnosis or treatment. Hence, there is a strong desire for a new treatment option with higher cure rate and good resistance barrier profile. In this randomized, double-blinded, placebo-controlled phase $2 / 3$ trial, we demonstrated the excellent efficacy of an interferon-

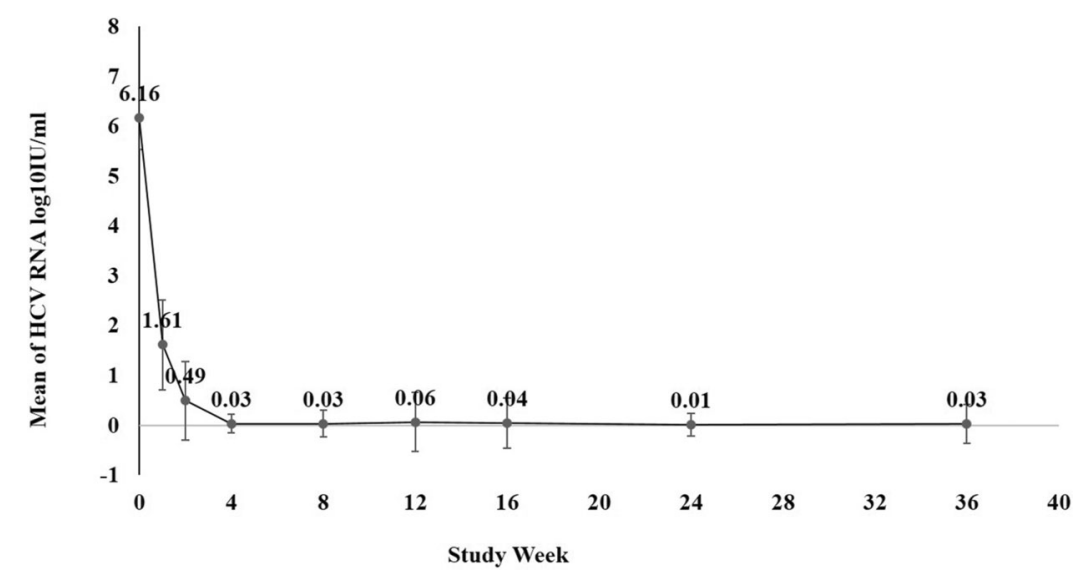

Fig. 3. Mean of HCV RNA $\log _{\mathbf{1 0}} \mathbf{I U} / \mathbf{m L}$ to the end of follow-up (per protocol set). When HCV RNA $<15$ IU/mL or reported as target not detected, HCV RNA was counted as $1 \mathrm{IU} / \mathrm{mL}$, or $\log _{10} 1 \mathrm{IU} / \mathrm{mL}$ in the Figure. 
Table 3. Baseline resistance-associated substitution analysis of HCV NS5A (per protocol set)

\begin{tabular}{lll}
\hline $\begin{array}{l}\text { Baseline with NS5A } \\
\text { resistance-Associated } \\
\text { species }\end{array}$ & $\begin{array}{l}\text { Treatment } \\
\text { arm, } n=309\end{array}$ & $\begin{array}{l}\text { Placebo arm, } \\
n=101\end{array}$ \\
\hline $\mathrm{R} 30 \mathrm{Q}, n(\%)$ & $38(12.3 \%)$ & $21(20.8 \%)$ \\
$\mathrm{Y} 93 \mathrm{H}, n(\%)$ & $21(6.8 \%)$ & $6(5.9 \%)$ \\
$\mathrm{R} 30 \mathrm{Q}+\mathrm{Y} 93 \mathrm{H}, n(\%)$ & $8(2.6 \%)$ & $1(1.0 \%)$ \\
$\mathrm{L} 28 \mathrm{M}, n(\%)$ & $3(1.0 \%)$ & $/$ \\
$\mathrm{R} 30 \mathrm{H}, n(\%)$ & $1(0.3 \%)$ & $/$ \\
$\mathrm{Y} 93 \mathrm{C}, n(\%)$ & $1(0.3 \%)$ & $/$ \\
$\mathrm{R} 30 \mathrm{Q}+\mathrm{L} 31 \mathrm{M}, n(\%)$ & $1(0.3 \%)$ & $/$ \\
$\mathrm{L} 28 \mathrm{M}+\mathrm{Y} 93 \mathrm{C}, n(\%)$ & $1(0.3 \%)$ & $/$ \\
$\mathrm{L} 28 \mathrm{M}+\mathrm{Y} 93 \mathrm{H}, n(\%)$ & $1(0.3 \%)$ & $/$ \\
$\mathrm{L} 28 \mathrm{Q}, n(\%)$ & $1(0.3 \%)$ & $1(1.0 \%)$ \\
Total, $n(\%)$ & $76(24.6 \%)$ & $29(28.7 \%)$ \\
\hline
\end{tabular}

free, all-oral antiviral therapy in treatment naïve, noncirrhosis, GT1 subjects, without resistance-associated species test before treatment initiation. The SVR12 rate in the treatment arm was $96 \%(306 / 318)$ in the FAS and $99 \%(306 / 309)$ the per protocol set models, respectively. The SVR12 for the placebo-controlled arm was 95\% (101/106) in the FAS and 99\% (100/101) the per protocol set sets.

In the interferon and RBV era, SVR was associated with several host-, viral- and drug-related characteristics, such as age, sex, baseline viral load, treatment duration, etc. ${ }^{8,9}$ Our results using subanalysis covering these factors showed there was little impact from age, gender, IL28B genotypes, and baseline viral load, and the efficacy was similarly high among all subgroups. The traditional negative predictors herein no longer delineate different response in SVR12 rates, whereas the potency of the investigational drug combination was demonstrated concordantly. The lower boundary of the $95 \%$ CI for the SVR12 rates in both arms were marginally higher than the historical control value (75\%) from the pegylated-interferon + RBV

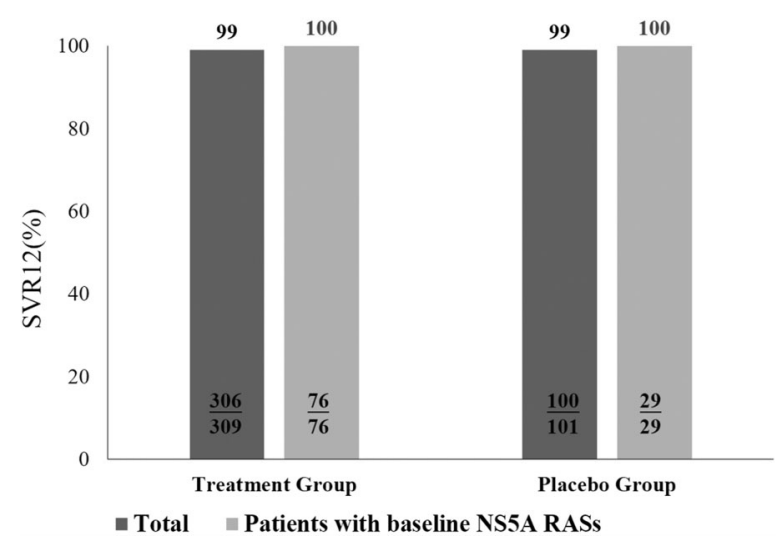

Fig. 4. Virological response of patients with baseline resistance-associated species.

Abbreviation: SVR12, Sustained virologic response at post-treatment week 12 . regimen, hence confirming the superiority hypothesis. Sensitivity analysis also reached a consistent conclusion.

Baseline resistance-associated substitutions (of resistance-associated species) in HCV NS5A are not uncommon in Chinese GT1b patients and may account for up to $19 \%$ prevalence with detrimental response in certain patients. ${ }^{10}$ Heretofore, the first generation NS5A inhibitors (such as daclatasvir, ledipasvir, and ombitasvir) have relatively lower NS5A resistance barriers and share cross-drug resistance, which undermines the efficacy of DAA therapies. ${ }^{11}$ According to drug labels and guidelines, the NS5A resistance-associated species test is recommended for elbasvir/ grazoprevir in GT1a, for daclatasvir plus asunaprevir in GT1b, and even for sofosbuvir/velpatasvir treatment in genotype 3 treatment-naive patients with cirrhosis and treatment-experienced patients (with or without cirrhosis). ${ }^{12}$ Nonetheless, consensus from clinicians is still the simplified testing as the preferable choice. Different from the NS5A inhibitors aforementioned, RDV is a pangenotypic HCV NS5A inhibitor with high resistance barrier and more potent antiviral activity in accordance with a higher administration dose at $200 \mathrm{mg}$ per day boosting to garner greater exposure. ${ }^{4}$ Besides, the wild-type HCV virus, conversely, in a previous study of RDV also demonstrated its potency to fully inhibit single NS5A substitution variants. ${ }^{13}$ In this study, GT1 NS5A resistance-associated species were detected in 76 of the participating subjects $(24.6 \%$, $76 / 309)$ in the treatment arm and 29 in the control arm (29\%, 29/101, per protocol set) at baseline (Fig. 2). All subjects with baseline NS5A resistance-associated species from both arms achieved SVR12. These results support a clinical use of this regimen without posing a baseline NS5A resistance-associated species test in the studied population, which was treatment naïve, noncirrhotic, and GT1 HCV subjects.

As to safety, the RDV + DNVr + RBV combination was well tolerated in the studied cohort in this trial. Most AEs were mild or moderate in intensity and were considered less relevant to investigational medications, which is concordant to results reported from the previous phase 2 studies using the same regimen. Severe AEs occurred in $2.8 \%$ (12/424) of the study population ( 7 in the treatment arm and 5 in the placebo arm), whereas none was assessed as related to study medication per discretion of the investigators. One patient died during the study due to a traffic accident and this event was considered not related to study drugs. One patient discontinued due to drug allergy. Most of the laboratory abnormalities in liver function were of mild to moderate severity (grade 1 and grade 2). Presence of the placebo arm in the study design hereby allowed for comparison of the safety profile with the active treatment arm. The resultant safety profile in the overall population was quite similar and comparable between the two arms, with exception of more anemia from the treatment arm which was not surprising due to the use of RBV.

In pharmacology, both RDV and danoprevir are pangenotypic anti-HCV inhibitors, ${ }^{4,5}$ possessing potent antiviral activities against HCV GT1 through genotype 6 species from in vitro and in vivo results. In phase 1 trials, RDV monotherapy showed potent antiviral activity and excellent safety profile in treatment-naïve subjects infected with GT1, genotype 2, and genotype $3 .^{12}$

The strength of this study is its being the first large scale, double-blinded, randomized, multicenter trial that confirms the efficacy and safety profiles of the new RDV + DNVr + RBV 
Xu X. et al: Efficacy of RDV + DNV regimen in GT1 CHC patients

Table 4. Adverse Events and Laboratory Abnormalities During the Double-Blinded Treatment Phase (FAS)

\begin{tabular}{lll}
\hline Patients, $n(\%)$ & Treatment arm, $n=318$ & Placebo arm, $n=106$ \\
\hline Any AEs & $298(94 \%)$ & $84(79 \%)$ \\
Treatment-related AEs leading to treatment discontinuation & $1(0.3 \%)^{*}$ & 0 \\
Any SAEs & $7(2 \%)^{\dagger}$ & $5(5 \%)^{\ddagger}$ \\
Treatment-related SAEs & 0 & 0 \\
Deaths & $1(0.3 \%)^{*}$ & 0 \\
Common adverse events ( $\geq 10 \%), n(\%)$ & & $5(5 \%)$ \\
$\quad$ Anemia $\quad$ Upper respiratory tract infection & $128(40 \%)$ & $22(21 \%)$ \\
$\quad$ Hemobilirubin rise & $67(21 \%)$ & $6(6 \%)$ \\
$\quad$ Alanine aminotransferase rise & $52(16 \%)$ & $3(2.83 \%)$ \\
$\quad$ Aspartate aminotransferase rise & $1(0.31 \%)$ & $2(1.89 \%)$ \\
$\quad$ Total bilirubin increase & 0 & 0 \\
\hline
\end{tabular}

* Discontinuation due to any adverse event occurred in one patient in the treatment group owing to drug allergy.

${ }^{\dagger}$ Serious adverse events in the treatment arm were vasovagal syncope, multiple bilateral rib fractures, died from a car accident. Hemorrhoids, cerebral infarction, infectious diarrhea, and community-acquired pneumonia, occurred in one patient each.

\$Serious adverse events in the placebo arm were acute exacerbation of chronic bronchitis, acute appendicitis and traumatic ciliary body detachment of the right eye, occurring in one patient each; left clavicle fracture and left third rib fracture in one; and, acute pelvic inflammatory disease and urinary tract infection in one.

\#One patient died due to traffic accident, and the event was not considered treatment-related.

Abbreviations: AEs, adverse events; SAEs, serious adverse events.

combination. The SVR12 of $99 \%$ regardless of pre-existing baseline NS5A resistance-associated species may provide a simple to use choice to clinicians in the defined study population. Good tolerability and safety profile further endorse application of this regimen in a large population. Nonetheless, this study may have some limitations. First, the use of RBV resulted in more anemia. However, most of the AEs were of mild or moderate severity and returned to normal after proper handling or intervention, as indicated from the trial results. The paradoxical role of RBV from the beneficial side may be eliminating quiescent viruses under other mechanisms apart from DAAs and reducing hepatocellular carcinoma incidence as reported before. The prolonged duration of using pegylated-interferon + RBV for 24 to 48 weeks preferably once plagued clinicians with safety concerns. Under this new combination, the duration was shortened to 12 weeks without interferon; therefore, the safety profile was very appreciable. Second, this study did not recruit cirrhosis subjects, due to insufficient proof of concept study results. We may look into the cirrhosis cohort further, including it in our future study design. Third, the use of ritonavir may trigger some potential concern in drug-drug interaction in clinical practice. Per the outcome of the safety results, there was limited discontinuation due to drug-drug interaction and little drug dose adjustment. This may be partially due to the observation that noncirrhosis patients are less sick and not taking complex concomitant comedications, while a shortened treatment duration of only 12 weeks may also help. Nevertheless, this may warrant a real-world observation to further understand the outcome.

In conclusion, treatment with all-oral RDV and DNVr in combination with RBV for 12 weeks resulted in SVR12 of $96 \%$ (FAS subgroup) and $99 \%$ (per protocol set subgroup) and was well tolerated in treatment-naïve noncirrhotic HCV GT1 Chinese patients.

\section{Acknowledgments}

Ascletis BioScience Co., Ltd. provided financial support for this study.

\section{Conflict of interest}

Lai Wei was consultant for AbbVie, Abbott, BMS, Gilead Sciences, Johnson \& Johnson, MSD, Novartis, and Roche, speaker for Abbott, BMS, Gilead Sciences, MSD, Novartis, Roche, and Ascletis, and received grant support from BMS and Roche. The other authors have no conflict of interests related to this publication.

\section{Author contributions}

Article conception and design (LW), acquisition of data (XX, $B F, Y G, S Z$, JS, XY, YM, YH, YK, XW, JL, YT, QH, QX, MW, PA, GG, HL, QN, RH, BN, WX, JZ, WH, YY, ML, YZ, YY, JJ, DY, LC, YY, YN, ZG, QZ, PH, FW, YL, DL, ZJ, JH, CC, and JJW), drafting and critical revision of the manuscript (LW).

\section{References}

[1] Global prevalence and genotype distribution of hepatitis $C$ virus infection in 2015: a modelling study. Lancet Gastroenterol Hepatol 2017;2:161-176. doi: $10.1016 /$ S2468-1253(16)30181-9.

[2] Rao H, Wei L, Lopez-Talavera JC, Shang J, Chen H, Li J, et al. Distribution and clinical correlates of viral and host genotypes in Chinese patients with chronic hepatitis C virus infection. J Gastroenterol Hepatol 2014;29:545-553. doi: 10.1111/jgh.12398.

[3] Ghany MG, Strader DB, Thomas DL, Seeff LB. Diagnosis, management, and treatment of hepatitis C: an update. Hepatology 2009;49:1335-1374. doi: $10.1002 /$ hep. 22759

[4] Lalezari JP, Farrell GC, Shah PS, Schwab C, Walsh D, Vig P, et al. PPI-668, a potent new pan-genotypic HCV NS5A inhibitor: Phase 1 efficacy and safety. Hepatology 2012; 56:1065A. 


\section{Xu X. et al: Efficacy of RDV + DNV regimen in GT1 CHC patients}

[5] Rajagopalan R, Misialek S, Stevens SK, Myszka DG, Brandhuber BJ, Ballard $J A$, et al. Inhibition and binding kinetics of the hepatitis $C$ virus NS3 protease inhibitor ITMN-191 reveals tight binding and slow dissociative behavior. Biochemistry 2009;48:2559-2568. doi: 10 . 1021/bi900038p.

[6] Seiwert SD, Andrews SW, Jiang Y, Serebryany V, Tan H, Kossen K, et al. Preclinical characteristics of the hepatitis $C$ virus NS3/4A protease inhibitor ITMN-191 (R7227). Antimicrob Agents Chemother 2008;52:4432-4441. doi: 10.1128/AAC.00699-08

[7] Kao JH, Yu ML, Chen CY, Peng CY, Chen MY, Tang H, et al. Twelve-week ravidasvir plus ritonavir-boosted danoprevir and ribavirin for non-cirrhotic HCV genotype 1 patients: A phase 2 study. J Gastroenterol Hepatol 2018; 33:1507-1510. doi: 10.1111/jgh.14096.

[8] Ghany MG, Nelson DR, Strader DB, Thomas DL, Seeff LB. An update on treatment of genotype 1 chronic hepatitis $C$ virus infection: 2011 practice guideline by the American Association for the Study of Liver Diseases. Hepatology 2011;54:1433-1444. doi: 10.1002/hep.24641.
[9] Petta S, Craxì A. How to optimize HCV therapy in genotype 1 patients: predictors of response. Liver Int 2013;33 Suppl 1:23-29. doi: 10.1111/liv.12053.

[10] Zhang Y, Cao Y, Zhang R, Zhang X, Lu H, Wu C, et al. Pre-existing HCV variants resistant to DAAs and their sensitivity to PegIFN/RBV in Chinese HCV genotype 1b patients. PLoS One 2016;11:e0165658. doi: 10 1371/journal.pone.0165658.

[11] Fujii H, Umemura A, Nishikawa T, Yamaguchi K, Moriguchi M, Nakamura $H_{\text {, }}$ et al. Real-world efficacy of daclatasvir and asunaprevir with respect to resistance-associated substitutions. World J Hepatol 2017;9:1064-1072. doi: $10.4254 /$ wjh.v9.i25.1064.

[12] Hepatitis C. guidance 2018 update: AASLD-IDSA recommendations for testing, managing, and treating hepatitis $C$ virus infection. Clin Infect Dis 2018;67:1477-1492. doi: 10.1093/cid/ciy585.

[13] Huang Q, Huang N, Huq A, Lau M, Peng E, Lalezari J, et al. Vast majority of detected NS5A resistant variants are not amplified in HCV patients during 3-day monotherapy with the optimized NS5A inhibitor PPI-668. J Hepatol 2013;58:S485. doi: 10.1016/S0168-8278(13)61193-9. 